\title{
Falla renal aguda por sobredosis de indometacina en un recién nacido prematuro con un ductus arterioso persistente
}

\author{
Dr. Augusto Winter G., Dr. José Luis Tapia I., Dr. Patricio Ventura-Juncá T., \\ Dra, Gabriela Jucz G., Dr. Walter Rivera M. ${ }^{1}$ \\ Indomethacin treatment for symptomatic patent ductus \\ arteriosus, drug overdose and renal failure.
}

A premature baby with symptomatic patent ductus arteriosus was treated with indomethacin. Due to an accidental overdose of the drug he developed acute renal failure that improved spontaneously after three days.

(Key words: patent ductus arteriosus; indomethacin therapy; renal failure).

El ductus arterioso persistente ha sido reconocido como una causa frecuente de descompensación cardiopulmonar y de compromiso respiratorio prolongado en prematuros durante la fase de recuperación de la enfermedad de membrana hialina ${ }^{1-4}$. Cuando el cortocircuito de izquierda a derecha a través del ductus es importante, se produce sobrecarga y eventualmente falla ventricular izquierda; esta falla ventricular izquierda y el cortocircuito de izquierda a derecha afectan desfavorablemente la resistencia vascular pulmonar, la distensibilidad pulmonar, la relación ventilación perfusión y la difusión e intercambjo gaseoso pulmonar $7,23,25$.

Es posible que la persistencia del ductus juegue algín papel en la génesis de hemorragia intracraneana, enterocolitis necrotizante, displa.

1. Departamento de Pediatría, unidad de neonatología. Hospital Clínico Pontificia Universidad Católica de Chile. sia broncopulmonar y perforación intestinal localizada11, 12, 15, 20.

Publicaciones recientes han demostrado que con el uso de indometacina se puede lograr eI cierre farmacológico del ductus arterioso $y$ debido a esto una disminución en la morbilidad de prematuros ${ }^{3-16}$.

Sin embargo, la droga puede provocar complicaciones serias como la que se relata a continuación.

\section{CASO CLINICO}

Varón de 37 semanas de edad gestacional que ingresó a la Unidad de Recién Nacidos del Hospital Clínico de la Universidad Católica por síndrome de dificultad respiratoria progresiva de instalación precoz con alteraciones radiológicas características de enfermedad de membrana hialina.

Fue tratado con presión positiva en la vía 
aérea y concentraciones de oxígeno de hasta $80 \%$ mejorando de la dificultad respiratoria. Al séptimo día de vida se auscultó un soplo sistólico 3/6 en el segundo espacio intercostal izquierdo, irradiado al dorso acompañado de pulsos saltones, hiperactividad ventricular izquierda, polipnea, taquacardia y signos radjológicos de edema pulmonar que no respondieron a la restricción del aporte hídrico y el empleo de diuréticos. Por esta razón, al noveno día de vida se indicó indometacina por vía endovenosa administrándose por error una dosis de $5 \mathrm{mg}$ por kilo, (veinticinco veces superior a la habitual), con desaparición inmediata del soplo ductal y las alteraciones de pulsos periféricos. Desde ese momento, el paciente presentó una oliguria mantenida durante 72 horas a pesar de la administra. ción de tres dosis de furosemida, sopor, edema periférico, y aumento de peso de $12 \%$ Los exámenes mostraton aumento progresivo de la creatinina plasmática hasta $4,4 \mathrm{mg} \mathrm{x}$ dl, y de nitrógeno urejco hasta $126 \mathrm{mg} \mathrm{x} \mathrm{dl}$, asociados con osmolaridad sérica de $280 \mathrm{~m}$ OsmxL y disminución del sodio sérico hasta $131 \mathrm{mEq} \times \mathrm{L}$. No hubo alteración en las concentraciones de potasio en el plasma.

Tratado con restricción de agua y electrolitos se recuperó progresivamente en 7 días, desce el momento del accidente. Controlado a los 21 dias de edad, su función renal era normal.

\section{DISCUSION}

Desde 1976 la indometacina ha sido utilizada para el cierre farmacológico del ductus arterioso persistente ${ }^{1}$. Inicialmente se empleó sólo por via oral y rectal en niños muy inmaduros con ductus persistente asociado a membrana hialina, pero en los últimos años con el uso intravenoso-se hall obtenido resultados iguales o mejores que con las otras rutas, en gran parte debido a la obtención de niveles plasmáticos adecuados, aún en niños muy inmaduros $9,15,16,20$.

Desde los inicios del empleo de indometacina han sido descritos efectos colaterales, entre ellos, la disfunción renal transitoria parece ser uno de los más frecuentes. Esta disfunción consiste en oliguria o anuria debida a disminución del flujo sanguíneo renal; disminución de la filtración glometular; reducción de la excreción fraccional de sodio; disminución de la depuración de agua libre y creatinina y aumento transitorio de las concentraciones plasmáticas de hormona antidiuréica, que explican la oliguria tan marcada ${ }^{8}$, $10,17,21,24$.

La supresión de la sintesis sistémica y renal de prostaglandinas y la reducción de su excreción producidas por la indometacina podrian ser contrarrestadas en parte por la administración de furosemida, que aumenta la excreción urinaria de prostaglandina $E$ pudiendo prevenir los efectos renales de ésta ${ }^{8-10}$. En este recién nacido, la administración de tres dosis sucesivas de furosemida no logró superar la oliguria relacionada con la sobredosis de indometacina.

Friedman y cols, en el año 1976, usaron en dos de sus pacientes dosis semejantes de indometacina igual a la que recibió nuestro paciente, pero por vía rectal, lo que podría haber produciđo concentraciones plasmáticas más bajas y por to mismo menor efecto sobre la función renal ${ }^{1}$.

A pesar de la duración y severidad de la oliguria y de las elevadas concentraciones de creatinina plasmática de este paciente, los niveles de potasio plasmático jamás llegaron a representar un riesgo para él. La naturaleza transitoria de la falla renal y su favorable evolución en el tiempo concuerda con lo señalado en otras publicaciones donde también la indometacina fue utilizada en dosis muy altas con efectos sólo transitorios sobre la función renal ${ }^{1}$.

\section{RESUMEN}

Un recién nacido prematuro con ductus arteroso persistente $y$ signos de insuficiencia cardiaca fue tratado accidentalmente con una sobredosis de indometacina endovenosa. El soplo de ductus desapareció pero inmediatamente después el paciente sufrió oliguria severa acompañada de retención nitrogenada y reducción de la concentración sérica de sodio, sin embargo evolucionó favorablemente recuperándose en 7 días sin mostrar evidencia de secuelas a la edad de 21 días.

\section{REFERENCIAS}

1. Friedman W., Hirschklau J., Printz M., Printz P., Kirk-Patrick S.+ Pharmacologic closure of patent ductus arteriosus in the premature infant. N. Engl. J. Med. 295: $\$ 26,1976$.

2. Yanagi $R$., Wilson A. er al: Indomethacin treatment for symptomatic patent ductus arteriosus: $A$ double-blind control study. Pediatrics 67: 647, 1981 .

3. Mahony L., Carnero V., Brett C., Heymann $M$., Clyman $R$.: Prophylactic indomethacin therapy for patent ductus arteriosus in very-low-birth weight infants. N. Engl, J, Med. 306: 507, 1982.

4. Yeh T.F., Luken I.A., Thalif A., Raval D., Carr I., Plldes R.S.: Intravenous indomethacin therapy in premature infants with persistent ductus aarteriosus: a double blind controlled study. J. Pediatr. 98; $137,1981$.

5. Yaffe $S$, Friedman W., Rogers D., Lang P., Ragrii M.. Saccar $C$.: The disposition of indomethacin in preterm babies. J. Pediatric 97: 10001, 1980. 
6. Thalji A., Corr I. et al: Pharmacokjnetics of intravenousty administered indomethacin in premature infants. J. Pediatric 97: 995, 1980.

7. Alierson D., Eldrigde $M$. et, al: Effect of patent ductus arteriosus on left ventricular output in premature infants. J. Pediatr. 102: 754, 1983.

8. Seyberth H., Roscher W., Hackental R., Wille L.: Effect of prolonged indomethacin therapy on renal function and selective vasoactive hormones in very low birth weight infants with symptomatic patent ductus arteriosus. J. Pediatr. 103:979, 1983.

9. Evans H., Bhat R. et al: A comparison of oral and intravenous indomethacin dispositions in the premature infant with patent ductus arteriosus. Ped. Pharmacology 1: 2S2, 1981.

10. Yeh $T$, Wilks $A$., Singh $J$, et at: Furosemide prevents the renal side effects of indomethacin therapy in premature infants with patent ductus arteriosus. J. Pediatt. 101: 433, 1982.

11. Merrit A., Horris $P$. et al: Early closure of the patent ductus arteriosus in very low birth weight infants: A controlled trial. J. Pediatr. 99: 281, 1981 .

12. Alpen G., Eyal F., Vinograd I., Udassin R., Amir G., Mogle P. Glick B.: Localized intestinal perforations after enteral administration of indomethacin in premature infants. J. Pediatr. 106: 277, 1985.

13. Mullet M., Croghan T., Myerberg D., Krall J., Neal W.: Indomethacin for vlosure of Patent Ductus Arteriosus in Prematures. Clin. Pediatr. 21: 217, 1982.

14. Heymann M., Rudolph A.. Silverman N.: Closure of the Ductus Arteriosus in premature infants by inhibition of prostaglandin synthesis. N. Engl. J. Med, 295: 530, 1976 .

15. Dudell $G$., Gersony W.: Patent ductus arteriosus in neonates with severe respiratory disease. $J$. Pediatr. 104: 915, 1984.

16. Obeyesekere H.. Pankhurst S., Yu U.: Plarmacological closure of the ductus arteriosus in preterm infants using indomethacin. Arch. D. Child. 55:
$271,1980$.

17. Usberti M., Pecoraro C., Federico S., Ciancioniso B., Guida B., Romano A., Grumetto L., Carbomaro L.: Mechanisms of action of indomethacin in tubular defects. Pediatrics 75: 501, 1985.

18. Petersen S., Christensen C., Jensen M., Ryssing E.: Serum indomethacin concentrations after intravenous administration to preterm infants with patent ductus arteriosus. Acta Pediatr. Scand. 70: 729, 1981.

19. Yeh T., Goldbarg H., Henek T., Thalji A., Pildens $R$. : Intravenous indomethacin therapy in premature infants with patent đuctus arteriosus. Am. J. Dis. Child. 136: 803, 1982.

20. Harris P.J., Merritt A., Alexson C., Longfield L., Manning $J_{, A}$.: Parenteral indomethacin for closure of the patent ductus arteriosus. An. J. Dis. Chidd. 136: $1005,1982$.

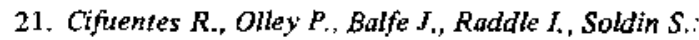
Indomethacis and renal function in premature infants with persistent ductus arteriosus. J. Pediatr. 95: 583,1979 .

22. Rudd P., Montananz P., Hallidie-Smith K., Silvermen $M .:$ Indomethacin treatment for patent ductus arteriosus in very low birth weight infants: double blind trial. Arch. Child. 58: 267, 1983.

23. Yeh T.F., Thalii A., Liken J., Raval D., Cart I., Fildes R.S: improved lung compliance after indomethacin therapy in premature infants with P.D.A. J. Pediatr. 96: 1112, 1980 (Abst.).

24. Betkerur M.V., Yeh T.F., Miller $K$., Classer R.J., Pildes R.S.: Indomethacin and its effects on renal function and urinary kallikrein excretion in premature infants with patent ductus arteriosus. Pediatrics 68: 99,1981

25. Nanly' C.M., Horn S. Conry J., Avery G:B.: Improved lung compliance after ligation of patent ductus arteriosus in hyaline membrane disease, $J$. Pediatr. 93: 682, 1978. 